\title{
In vitro damage of Candida albicans biofilms by chitosan
}

\author{
YU PU ${ }^{1}$, AIBO LIU ${ }^{1}$, YUQIANG ZHENG ${ }^{2 *}$ and BIN YE ${ }^{1,3^{*}}$ \\ ${ }^{1}$ Department of Pathogenic Biology, Chongqing Medical University, Chongqing 400016; \\ ${ }^{2}$ Department of Medicine Laboratory, Children's Hospital of Chongqing Medical University, Chongqing 400014; \\ ${ }^{3}$ Research Center for Molecule Medicine and Tumor, Chongqing Medical University, Chongqing 400016, P.R. China
}

Received February 2, 2014; Accepted June 10, 2014

DOI: $10.3892 / \mathrm{etm} .2014 .1839$

\begin{abstract}
With the increasing usage of indwelling medical devices in clinical practice, the frequency of fungal infections has increased, such as that of Candida albicans (C. albicans). Biofilms, a protected niche for microorganisms, are resistant to a range of current antifungal agents. Chitosan is a polyatomic biopolymer with advantageous biocompatibility, biodegradation, nontoxicity and antibacterial properties. To investigate the inhibitory effect of chitosan on biofilms formed by C.albicans, cell viability, 2,3-bis(2-methoxy-4-nitro-5-sulfophenyl)2H-tetrazolium-5-caboxanilide reduction, and morphological assays, including fluorescence microscopy and scanning electron microscopy (SEM), were employed. As assessed by cell viability assay, chitosan showed significant inhibitory effects on the planktonic cells and the biofilm of $C$. albicans in a dose-dependent manner. Fluorescence microscopy and SEM assays confirmed that the chitosan-treated group showed delayed C.albicans biofilm formation with defect morphological features, due to the inhibitory effects of the vast majority of fungal cell growth. In conclusion, $C$. albicans biofilms were compromised by the treatment with chitosan, providing an alternative therapeutic strategy against the fungal biofilms in the medical devices.
\end{abstract}

\section{Introduction}

Candida albicans (C. albicans), an opportunistic pathogen, is able to attack hosts that are immunocompromised or

Correspondence to: Mr. Yuqiang Zheng, Department of Medicine Laboratory, Children's Hospital of Chongqing Medical University, No. 136 Zhong Shan Er Road, Yuzhong, Chongqing 400014, P.R. China

E-mail: zheng_yuqiang@cqmu.edu.cn

Professor Bin Ye, Department of Pathogenic Biology, Chongqing Medical University, No. 1 Yi Xue Yuan Road, Yuzhong, Chongqing 400016, P.R. China

E-mail: yebina@sohu.com

${ }^{*}$ Contributed equally

Key words: Candida albicans, chitosan, biofilms otherwise debilitated. With the increased use of immunosuppressive and cytotoxic drugs, antibiotic abuse and implanted devices, including urinary catheters and endotracheal tubes, the prevalence of fungal infections has also increased (1-3). C. albicans was the leading cause of pulmonary fungal infection in 2011 and the drug resistance rate demonstrates a tendency to increase year by year (4). A number of implanted devices, including intravascular or urinary catheters and endotracheal tubes, are associated with fungal infections, and a biofilm may be detected on their surface (5-7). In recent years, emerging cases of $C$. albicans drug resistance have been primarily attributed to the formation of biofilms, with resistance increasing in conjunction with the maturation of the biofilm (8). Mature Candida biofilms consist of a complex three-dimensional structure of layers of yeast cells, hyphae and an abundant exopolysaccharide matrix $(9,10)$. As natural resistance barriers of antifungal drugs, biofilms play a significant role in the invasion and dissemination of C.albicans.

Chitosan is a natural linear polyatomic biopolymer comprising $\mathrm{N}$-acetyl-D-glucosamine and $\beta$ - $(1,4)$-linked $D$-glucosamine. A previous study revealed that surfaces coated with chitosan were able to resist biofilm formation by bacteria and fungi in vitro (11). Chitosan is nontoxic to humans and exhibits excellent biocompatibility. The biopolymer is easily catalyzed to oligosaccharides by various biological enzymes, and is considered biodegradable (12); thus, can be absorbed by the body. Previous studies have demonstrated that chitosan not only has immunoregulatory efficacy, but also a naturally broad spectrum of antibacterial activity that enables the inhibition of biofilm formation $(11,13,14)$.

Chitosan is an extensively studied biomacromolecule that is typically derived from chitin, a major component of crustacean shells. Due to their biocompatible and biodegradable properties, chitosan and its derivatives have been proposed for application in novel drug studies. The effect of chitosan on bacterial biofilms has been extensively reported; however, the aim of the present study was to investigate the susceptibility of $C$. albicans biofilms to chitosan, with an emphasis on determining the effects of chitosan on various biofilm growth phases and architectural organization.

\section{Materials and methods}

C. albicans. A C. albicans strain, previously isolated from a patient, was obtained from the Clinical Laboratory of 
the Children's Hospital of Chongqing Medical University (Chongqing, China). The fungus was inoculated on a chocolate agar plate and grown at $37^{\circ} \mathrm{C}$ for $48 \mathrm{~h}$. A single colony of the desired strain was inoculated into $8 \mathrm{ml}$ Sabouraud dextrose broth (SDB; Sangon Biotech Co., Ltd., Shanghai, China) and incubated overnight at $37^{\circ} \mathrm{C}$ in a rotary shaker at $220 \mathrm{rpm}$.

Chitosan susceptibility in C. albicans planktonic cells. Chitosan (Sigma-Aldrich, St. Louis, MO, USA) was solubilized in $0.2 \%$ acetic acid at a working concentration of $1 \%$ to $\mathrm{pH}$ 5.0-5.5. Serial two-fold dilutions of chitosan stock solutions in SDB were prepared in plastic centrifuges over a range of $0.0078-0.5 \%$ and stored at $4^{\circ} \mathrm{C}$. A standard inoculum of $1 \times 10^{7}$ cells [optical density $(\mathrm{OD})_{600}$ ] from the overnight culture of the fungal strain was prepared prior to each experiment. Briefly, $100 \mu 1$ C. albicans planktonic cells were mixed with $100 \mu \mathrm{l}$ chitosan solution of various concentrations and incubated for $48 \mathrm{~h}$ at $37^{\circ} \mathrm{C}$. Data were obtained from three independent experiments. For the positive control, C. albicans planktonic cells were incubated in the presence of $200 \mu \mathrm{l}$ SDB without chitosan, while in the negative control, $200 \mu 1 \mathrm{SDB}$ only was incubated in wells under otherwise identical conditions. The minimum inhibitory concentrations $(\mathrm{MIC})_{50}$ for the biofilms and planktonic cells were defined as the minimum antifungal concentration that caused $\geq 50 \%$ fungal damage compared with the untreated controls.

Measurement of chitosan susceptibility in biofilms using a 2,3-bis-(2-methoxy-4-nitro-5-sulfophenyl)-2H-tetrazolium-5carboxanilide (XTT) reduction assay. C. albicans biofilm experiments were performed in untreated 96-well plates. The wells of the 96-well plates were incubated for various time periods $(2,8,24$ and $48 \mathrm{~h})$ in $100 \mu \mathrm{l} C$. albicans suspension $\left(1 \times 10^{7}\right.$ cells $\left./ \mathrm{ml}\right)$ at $37^{\circ} \mathrm{C}$. The medium in each well was removed at the indicated time points and the biofilms were washed twice with phosphate-buffered saline (PBS). Following the removal of PBS, $100 \mu \mathrm{l}$ chitosan solution of various concentrations were added to one well of a 96-well plate and then incubated for additional $48 \mathrm{~h}$ at $37^{\circ} \mathrm{C}$. A formazan salt-based XTT reduction assay was performed to assess the metabolic activity (15). All the tests were performed in duplicate and the average was calculated. Positive and negative controls were established as previously described. A total of $100 \mu \mathrm{l}$ XTT-menadione solution, consisting of XTT (Sangon Biotech) salt solution $(0.5 \mathrm{~g} / 1$ in Ringer-Locke liquor) mixed with menadione (Sangon Biotech Co., Ltd.) solution (1 mM in acetone; Sigma-Aldrich), was added to each well. The 96-well plates were incubated for $2 \mathrm{~h}$ at $37^{\circ} \mathrm{C}$. Colorimetric changes were scanned at $490 \mathrm{~nm}$ with a Varioskan ${ }^{\text {TII }}$ Flash Multimode Reader (Thermo Fisher Scientific, Waltham, MA, USA).

C. albicans biofilm formation with cover slips. Cover slips $(0.8 \mathrm{x} 0.8 \mathrm{~cm})$ that were used for biofilm growth were soaked in concentrated sulfuric acid overnight. The following day, the concentrated sulfuric acid was washed with flowing water and the clean cover slips were immersed in $95 \%$ alcohol overnight. All the cover slips were washed three times with deionized water. For biofilm growth on the treated cover slips, as aforementioned, the cover slips were placed in 24-well plates and immersed in fetal bovine serum (FBS; Sigma-Aldrich, Beijing,
China) at $4^{\circ} \mathrm{C}$ overnight. Following this pretreatment, the cover slips were washed with PBS $(0.01 \mathrm{M})$ to remove the residual FBS. To ensure uniform biofilm formation, the cover slips were immersed in $1 \mathrm{ml}$ standardized cell suspension $\left(1 \times 10^{7}\right.$ cells $\left./ \mathrm{ml}\right)$ and incubated for $90 \mathrm{~min}$ at $37^{\circ} \mathrm{C}$. The cover slips were lifted carefully using tweezers and gently placed in each well of the 24-well plate containing $1.5 \mathrm{ml}$ fresh SDB medium. Samples were incubated for various durations at $37^{\circ} \mathrm{C}$.

Susceptibility of the biofilms to chitosan. To evaluate the chitosan susceptibility of $C$. albicans cells grown in developing biofilms, the pretreated cover slips were immersed in $1 \mathrm{ml}$ standardized cell suspension $\left(1 \times 10^{7}\right.$ cells $\left./ \mathrm{ml}\right)$ and incubated for $90 \mathrm{~min}$ at $37^{\circ} \mathrm{C}$. The cover slips were lifted carefully using tweezers and gently placed in each well of the 24-well plate containing $1 \mathrm{ml}$ fresh SDB medium. A $0.0625 \%$ chitosan solution was added to 24 -well plates with cover slips and incubated for an additional 8,24 and $48 \mathrm{~h}$ at $37^{\circ} \mathrm{C}$.

Fluorescence microscopy. Cover slips pretreated with biofilms were transferred to microscope slides and stained for $1 \mathrm{~min}$ with $50 \mu \mathrm{l}$ Calcofluor White M2R [0.05\% (vol/vol); Sigma-Aldrich] under minimal ambient light or in a darkroom. The biofilms were observed immediately under an ultraviolet (UV) range of $440 \mathrm{~nm}$ excitation and 500-520 nm emission wavelengths. Any superfluous fluorochrome on the cover slips was absorbed by the filter paper. The stained biofilms were examined under a fluorescence microscope.

Scanning electron microscopy (SEM). C. albicans biofilms were grown on pretreated cover slips in 24-well plates, as described previously. In the chitosan group, $C$. albicans biofilms were incubated in $0.0625 \%$ chitosan. For the positive control, C. albicans biofilms were incubated in the presence of $200 \mu \mathrm{l} \mathrm{SDB}$ without chitosan for $24 \mathrm{~h}$. The cover slips with biofilms were subsequently washed three times with PBS and transferred to an additional 24-well plate containing 2.5\% glutaraldehyde at $4^{\circ} \mathrm{C}$. The samples were prepared using a regular method for electron microscopy examination (16) and viewed under an S-3000N scanning electron microscope (Hitachi High-Technologies, Tokyo, Japan). Two separate sets of culture were prepared.

Statistical analysis. Statistical analyses were performed using SPSS 19.0 software (SPSS, Inc., Chicago, IL, USA). All data were tested for normality and transformed when necessary to meet the assumption of normal distribution. P-values were calculated by one-way analysis of variance and Fisher's least significant difference test was conducted to determine differences among the test groups. $\mathrm{P}<0.05$ was considered to indicate a statistically significant difference.

\section{Results}

MIC values of chitosan on C. albicans planktonic cells. Fig. 1 shows the results of the experiment assessing the effect of chitosan at various concentrations on C. albicans planktonic cell growth. Chitosan, at a concentration of $>0.0313 \%$, was shown to severely inhibit the activity of $C$. albicans planktonic cells grown for $24 \mathrm{~h}$. The OD decreased significantly when 


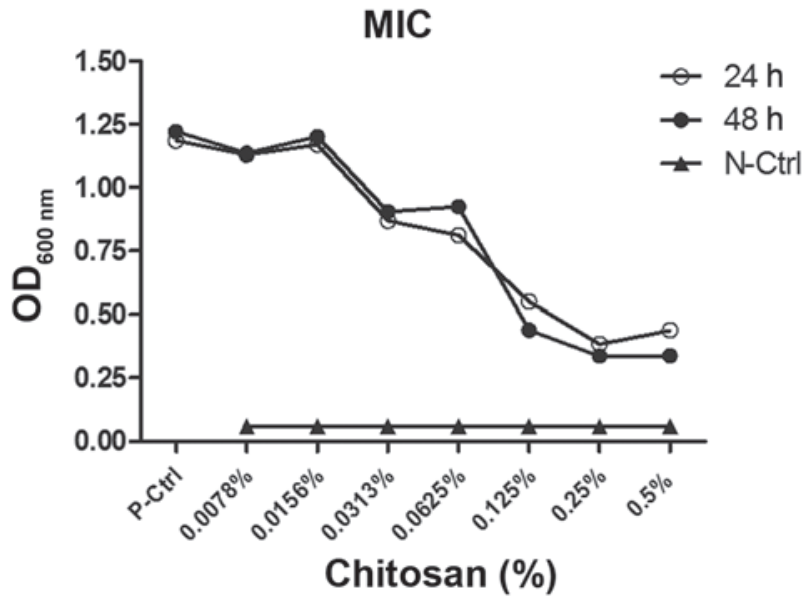

Figure 1. Growth curve of Candida albicans (C. albicans) planktonic cells with chitosan. Planktonic cells were co-incubated with various concentrations $(0.5,0.25,0.125,0.062,0.0313,0.0156$ and $0.0078 \%)$ of chitosan for 24 and $48 \mathrm{~h}$. Sabouraud dextrose broth served as a negative control. The line curves show that chitosan $(>0.0313 \%)$ strongly inhibited the growth of C. albicans. Experiments were conducted three times, with similar results each time. OD, optical density; MIC, minimum inhibitory concentration.

compared with the positive control $(\mathrm{P}<0.05)$. When comparing the effect of chitosan over 24 and $48 \mathrm{~h}$, the MIC of chitosan exhibited no statistically significant change on cell growth.

C. albicans biofilm formation. An XTT reduction assay was used to quantify the effects of chitosan on biofilms produced by $C$. albicans. As shown in Fig. 2, the different phases of C. albicans biofilm formation were significantly susceptible to chitosan. At $2 \mathrm{~h}$, the OD values, as determined by an enzyme-labeled instrument, were similar to when the concentration of chitosan was $>0.0313 \%$. A statistically significant difference was observed in the OD values between the 0.0313 and $0.0156 \%$ chitosan groups $(\mathrm{P}<0.05)$. In addition, a marked difference was observed between all the chitosan groups and the positive control $(\mathrm{P}<0.05)$. The results did not demonstrate a statistically significant difference between biofilm development at 2 and $8 \mathrm{~h}$, and the optimum concentration of chitosan was almost equal. Compared with the biofilms grown for 2 or $8 \mathrm{~h}$, the OD values for the biofilms grown for 24 or $48 \mathrm{~h}$ were significantly higher following exposure to chitosan at a concentration of $0.0313 \%$. The results revealed that biofilms in the mature phase (24-8 h) demonstrated less susceptibility to higher concentrations of chitosan compared with those in the early phase (2-8 h). Thus, biofilm formation exhibits a significant resistance to the antifungal activity of chitosan.

A previous study observed that $C$. albicans biofilm formation on polymethylmethacrylate strips progresses in three distinct developmental phases: Early, intermediate and maturation (10). The effect of chitosan on the temporal development of $C$. albicans biofilms on cover slips was investigated with fluorescence microscopy using Calcofluor White M2R, a UV-excitable dye that binds chitin and $\beta$-glucan and has long been used to highlight the fungal cell wall (17). Fluorescence microscopy was performed to visualize and compare the three distinct developmental phases between the untreated and chitosan-treated C. albicans biofilms (Fig. 3). In the early stage, characteristic microcolonies and short hyphae appeared
A

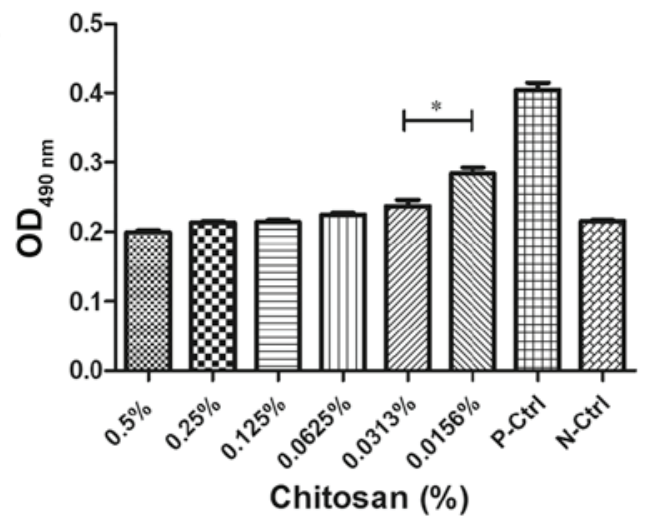

B

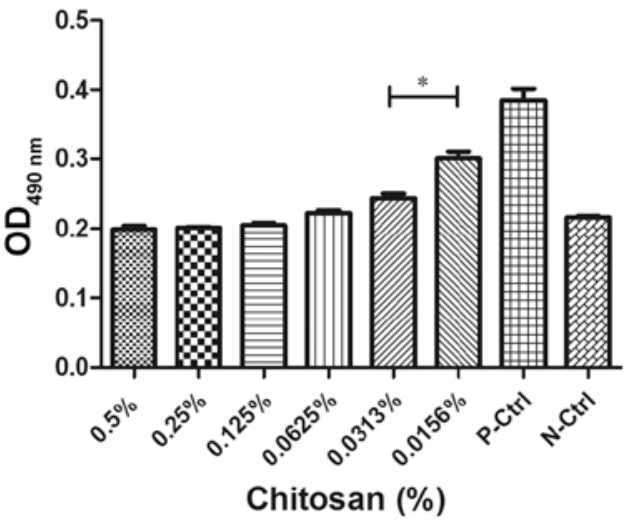

C

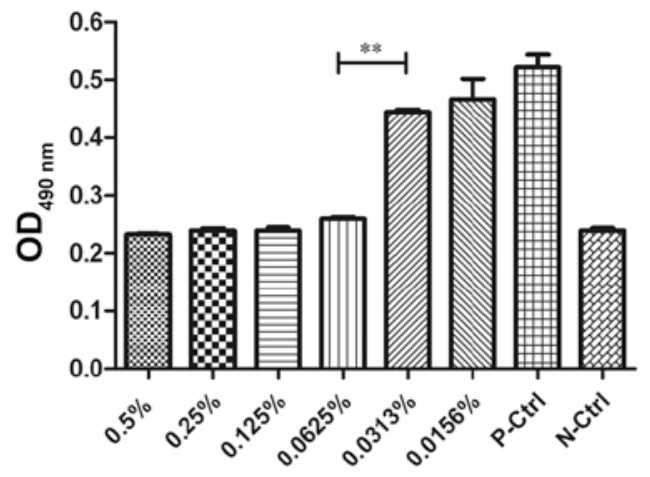

Chitosan (\%)

D

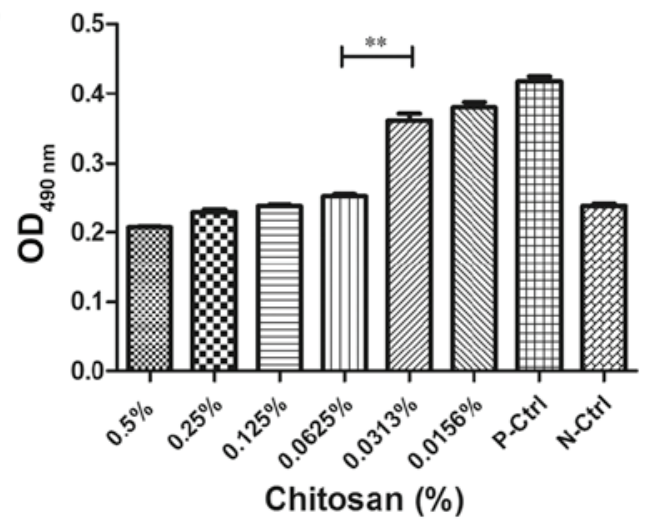

Figure 2. Candida albicans (C. albicans) biofilm development with chitosan resistance. The susceptibility of $C$. albicans biofilms, incubated for (A) $2 \mathrm{~h}$, (B) $8 \mathrm{~h}$, (C) $24 \mathrm{~h}$ and (D) $48 \mathrm{~h}$, to various concentrations $(0.5,0.25,0.125$, $0.0625,0.0313$ and $0.0156 \%$ ) of chitosan are represented as histograms. For the positive control, C. albicans planktonic cells were incubated without chitosan; for the negative control, sabouraud dextrose broth only was incubated in the wells. The OD values at the various stages of biofilm development were compared with those of the fungal cells and the negative control. Experiments were performed twice, with similar results obtained each time. OD, optical density. 
A

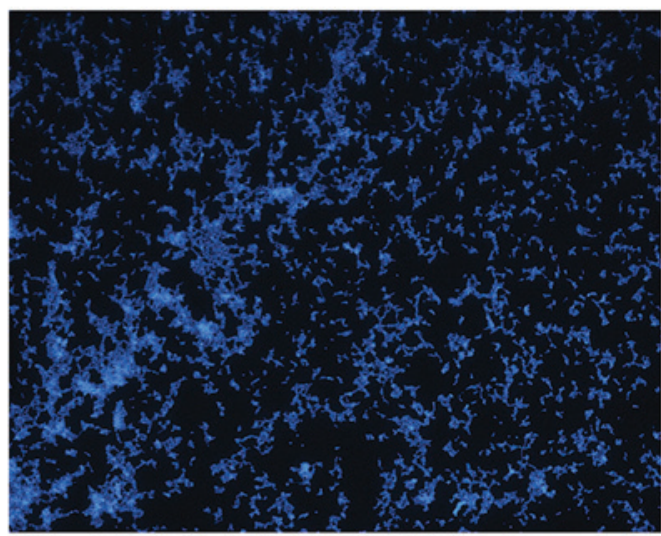

C

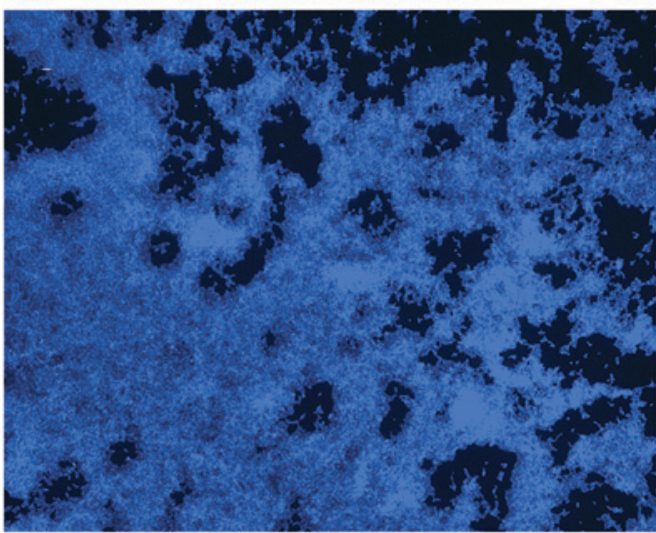

E

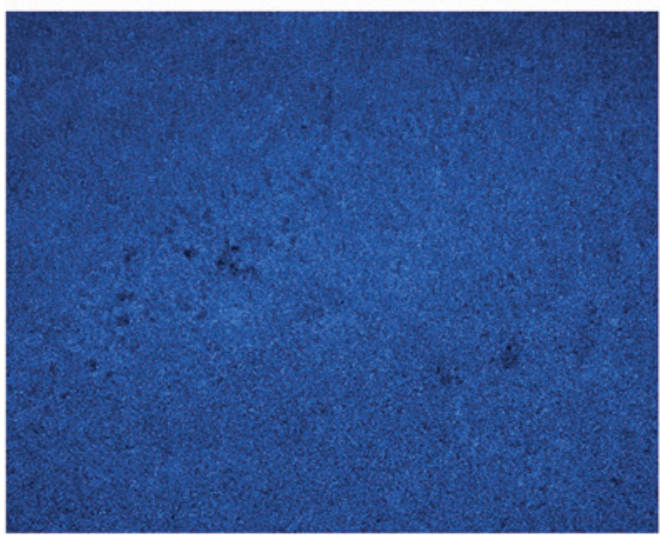

B

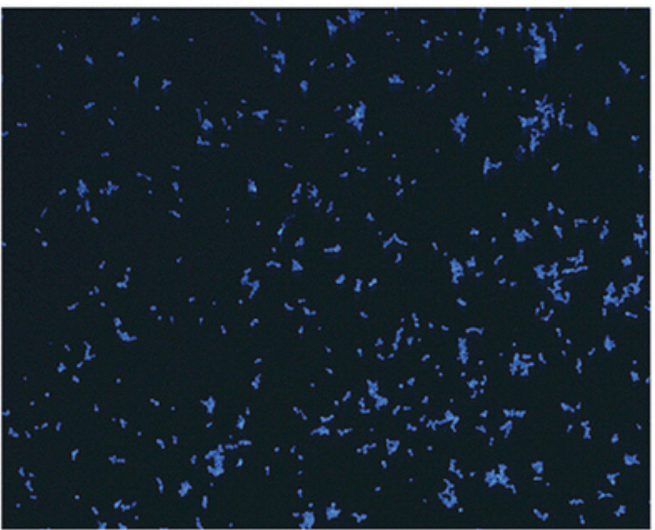

D

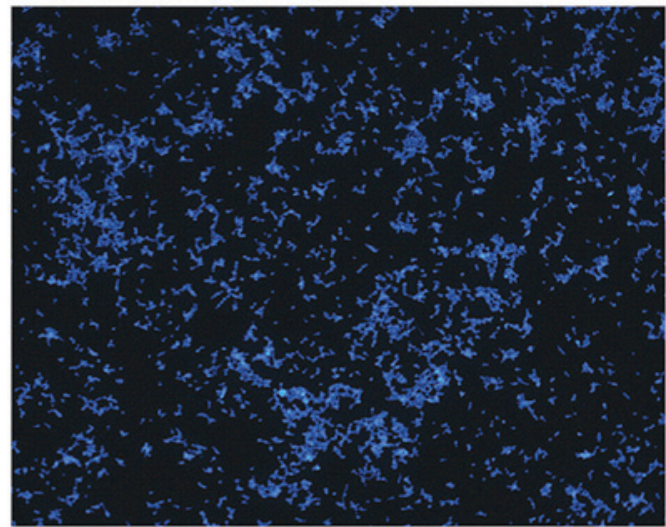

F

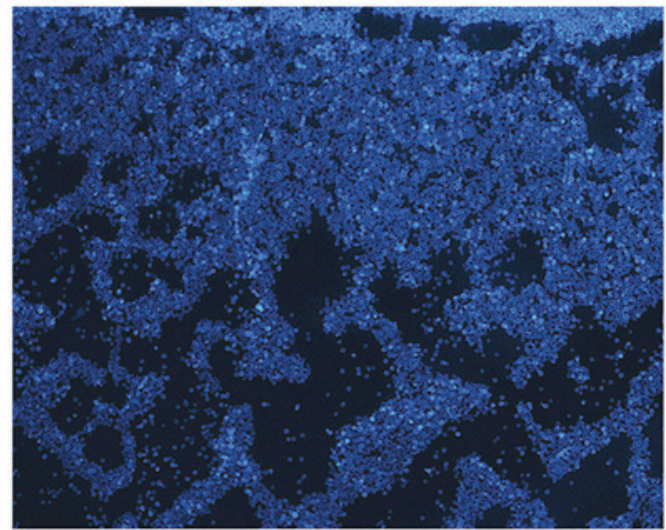

Figure 3. Fluorescence microscopy images showing the three developmental phases of Candida albicans biofilms in the (A, C and E) absence or (B, D and $\mathrm{F}$ ) presence of $0.0625 \%$ chitosan (A and B, early phase; $\mathrm{C}$ and $\mathrm{D}$, intermediate phase; $\mathrm{E}$ and $\mathrm{F}$, maturation phase; magnification, $\mathrm{x} 10$ ).

on the surface of the cover slips of the untreated cells (Fig. 3A). As shown in Fig. 3B, a few yeast cells were dispersed in the $0.0625 \%$ chitosan-treated group at the same stage. During the intermediate developmental phase, a dense network of yeast cells surrounded by a large amount of noncellular material was observed in the untreated group, with the metabolically active cells existing in a network (Fig. 3C). The quantity of yeast cells in the chitosan-treated group at the same stage was lower compared with the untreated group, and the cells were unable to congregate and develop into a bioflim (Fig. 3D). In the maturation development phase, the surfaces of the cover slips were coated with a thick layer of yeast cells and noncellular material; however, it was difficult to distinguish between the cell types (Fig. 3E). By contrast, the chitosan-treated group exhibited yeast cells lacking a network structure and a significantly lower metabolic activity compared with the untreated group (Fig. 3F).
Fluorescence microscopy images were used to correlate the XTT reduction assay results with the visual effects of the biofilm formation at the various exposure times. The results indicated that chitosan was not only able to inhibit the metabolic activity of cells in maturing biofilms, but also delay biofilm formation by terminating the fusion process.

Direct visualization of the effect of chitosan on C. albicans biofilms. SEM examination was used to visualize the structural differences between normal and chitosan-treated C. albicans biofilms. Normal C. albicans biofilms exhibited a dense network of hyphae surrounded by vast amounts of exopolymeric matrix (Fig. 4A). By comparison, chitosan-treated C. albicans expressed a biofilm that lacked a normal network structure and released polysaccharide (Fig. 4B). These results demonstrated that chitosan significantly inhibited C. albicans biofilms in vitro. 

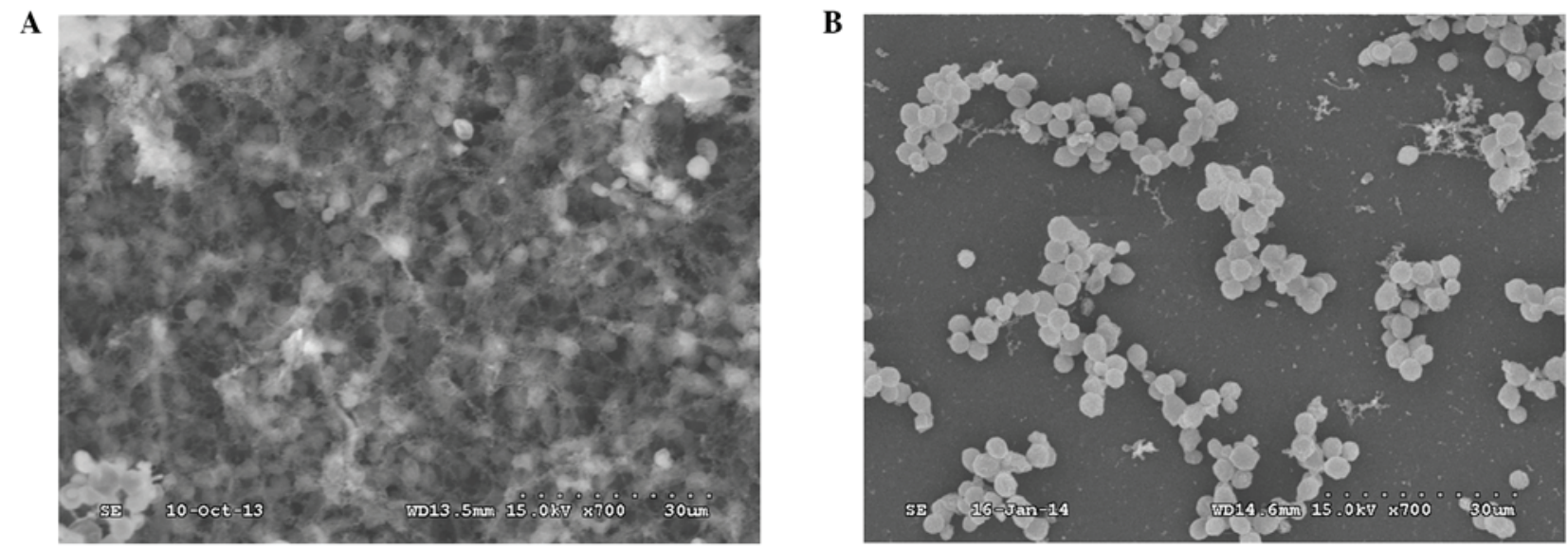

Figure 4. (A) Candida albicans biofilms cultured in sabouraud dextrose broth for 24 h showed fungal cells surrounded by large amounts of exopolymeric matrix. (B) By contrast, biofilms co-incubated with $0.0625 \%$ chitosan exhibited yeast cells without a capsule, releasing polysaccharides. Scale bar, $30 \mu \mathrm{m}$. Maginification, x700.

\section{Discussion}

Currently, fungal infections present an increasing threat to the growing number of immunocompromised patients. These contaminations, of which Candidiasis is the most common, represent one of the most prevalent infections in US hospitals (18). A leading causative agent of Candidiasis continues to be $C$. albicans (18). However, numerous problems remain in the current therapies for fungal infections. Antifungal treatment strategies for C. albicans are limited to a small armamentarium of compounds, which mainly include azoles (such as fluconazole), polyenes (such as amphotericin B) and echinocandins (19). However, there are clinical drawbacks to these drugs due to their toxicity (20). Therefore, identifying alternative antifungal agents with fewer side effects is required. Furthermore, since the majority of fungal infections are caused by biofilms, this provides an additional reason for the low success rate of treatment. Chitosan offers a flexible, biocompatible platform for designing coatings to protect surfaces from infection and decreasing the metabolic activity and survival rate of Candida species biofilms (21). The characteristics of chitosan, including its antiadherent and antifungal properties, mean that it is a strong candidate for treatment against fungal biofilms. In a normal conjunction, the $C$. albicans biofilm proceeds into early, intermediate and maturation phases of development. The extracellular material, as observed by microscopy, is predominantly composed of cell wall-like polysaccharides containing mannose and glucose residues. Biofilms grown on cover slips have a distinct biphasic structure composed of an adherent blastospore layer covered by sparser hyphal elements embedded in a deep layer of extracellular material. The structure that meditates cell interactions with the environment is the cell wall, which may be a viable factor in the adhesion of fungi to solid surfaces.

The present study analyzed the role of chitosan on C. albicans using the MIC. The results indicated that chitosan had high fungistatic activity against these planktonic cells Repeated XTT reduction assay analysis revealed that chitosan at a concentration of $0.0313 \%$ had the potential to kill $>50 \%$ of cells in the early and intermediate phases of biofilm development. However, a higher concentration of chitosan was required to kill cells in mature biofilms. Mature biofilms of C. albicans were significantly less susceptible to chitosan than planktonic cells and initial biofilms. The results of the current study indicated that an addition of exogenous chitosan to $C$. albicans biofilms reduced the metabolic activity of cells significantly and apprehended the adhesion of yeast cells to the polystyrene surface. Previous studies have demonstrated that the biofilm phenotype confers resistance to antifungal therapy, which is consistent with the results of the present study $(22,23)$. Physical stress of the biofilm structure due to permeabilization of the cellular membrane, which permits higher levels of penetration by chitosan and a more effective delivery of its antifungal activity, may be the cause of this phenomenon $(24,25)$.

Fluorescence and SEM techniques allowed the morphology and structure of biofilms to be analyzed in the present study. Fluorescence microscopy visualized the gross biofilm morphology and the appearance of the extracellular matrix during biofilm formation. SEM visualized the structure of the biofilms surface without distortion of the native biofilm structure. Progressing in three distinct developmental phases, C. albicans biofilm formation is divided into early, intermediate and maturation stages. The results of the present study revealed that in the overall biofilm formation period, the chitosan-treated biofilms exhibited a slower growth compared with those that were untreated. Although chitosan treatment did not completely destroy the yeast cells, the staining observed with fluorescence and SEM demonstrated that chitosan-treated biofilms were significantly inhibited in the formation of a complex network.

In conclusion, the results of the present study demonstrated that chitosan may be developed as an antimicrobial agent against the treatment of clinically-associated fungal biofilm diseases. Although an inhibitory effect of chitosan on biofilm production by $C$. albicans was reported in the laboratory and in clinical isolates in vitro, further studies using animal models of pulmonary $C$. albicans infection are required. 


\section{Acknowledgements}

The study was supported in part by grants from the National Natural Science Foundation of China for Young Scholars (no. 81000745) and the Project of Medicine Science of Health Bureau of Chongqing [nos. (2009)66 and (2010)51]. The authors thank the Clinical Laboratory at the Children's Hospital of Chongqing Medical University for providing the C. albicans strain.

\section{References}

1. Hallahan AR, Shaw PJ, Rowell G, O'Connell A, Schell D and Gillis J: Improved outcomes of children with malignancy admitted to a pediatric intensive care unit. Crit Care Med 28: 3718-3721, 2000.

2. Booy R, Habibi P, Nadel S, et al: Reduction in case fatality rate from meningococcal disease associated with improved healthcare delivery. Arch Dis Child 85: 386-390, 2001.

3. Tenner PA, Dibrell H and Taylor RP: Improved survival with hospitalists in a pediatric intensive care unit. Crit Care Med 31: 847-852, 2003

4. Chen Yan-jun WF: Study on pathogens causing lower respiratory tract infections and their drug resistance. Zhong Guo Bing Yuan Sheng Wu Xue Za Zhi 8: 561-563, 2013 (In Chinese).

5. Adair CG, Gorman SP, Feron BM, et al: Implications of endotracheal tube biofilm for ventilator-associated pneumonia. Intensive Care Med 25: 1072-1076, 1999.

6. Crump JA and Collignon PJ: Intravascular catheter-associated infections. Eur J Clin Microbiol Infect Dis 19: 1-8, 2000.

7. Maki DG and Tambyah PA: Engineering out the risk for infection with urinary catheters. Emerg Infect Dis 7: 342-347, 2001.

8. Douglas LJ: Candida biofilms and their role in infection. Trends Microbiol 11: 30-36, 2003

9. Blankenship JR and Mitchell AP: How to build a biofilm: a fungal perspective. Curr Opin Microbiol 9: 588-594, 2006.

10. Chandra J, Mukherjee PK and Ghannoum MA: In vitro growth and analysis of Candida biofilms. Nat Protoc 3: 1909-1924, 2008

11. Carlson RP, Taffs R, Davison WM and Stewart PS: Anti-biofilm properties of chitosan-coated surfaces. J Biomater Sci Polym Ed 19: 1035-1046, 2008.

12. Alonso MJ and Sánchez A: The potential of chitosan in ocular drug delivery. J Pharm Pharmacol 55: 1451-1463, 2003.
13. Kulikov SN, Tiurin IuA, Fassakhov RS and Varlamov VP: Antibacterial and antimycotic activity of chitosan: mechanisms of action and role of the structure. Zh Mikrobiol Epidemiol Immunobiol 5: 91-97, 2009 (In Russian).

14. Pasquantonio G, Greco C, Prenna M, et al: Antibacterial activity and anti-biofilm effect of chitosan against strains of Streptococcus mutans isolated in dental plaque. Int J Immunopathol Pharmacol 21: 993-997, 2008.

15. Meshulam T, Levitz SM, Christin L and Diamond RD: A simplified new assay for assessment of fungal cell damage with the tetrazolium dye, $(2,3)$-bis-(2-methoxy-4-nitro-5-sulphenyl)-(2H)-tetrazolium-5-carboxanilide (XTT). J Infect Dis 172: 1153-1156, 1995.

16. Tsang PW, Bandara HM and Fong WP: Purpurin suppresses Candida albicans biofilm formation and hyphal development. PLoS One 7: e50866, 2012.

17. Albani JR and Plancke YD: Interaction between calcofluor white and carbohydrates of alpha 1-acid glycoprotein. Carbohydr Res 314: 169-175, 1998.

18. Wisplinghoff H, Ebbers J, Geurtz L, Stefanik D, Major Y, Edmond MB, Wenzel RP and Seifert H: Nosocomial bloodstream infections due to Candida spp. in the USA: species distribution, clinical features and antifungal susceptibilities. Int J Antimicrob Agents 43: 78-81, 2014.

19. Georgopapadakou NH and Walsh TJ: Human mycoses: drugs and targets for emerging pathogens. Science 264: 371-373, 1994.

20. Ramage G, VandeWalle K, Bachmann SP, Wickes BL and López-Ribot JL: In vitro pharmacodynamic properties of three antifungal agents against preformed Candida albicans biofilms determined by time-kill studies. Antimicrob Agents Chemother 46: 3634-3636, 2002.

21. Martinez LR, Mihu MR, Tar M, et al: Demonstration of antibiofilm and antifungal efficacy of chitosan against candidal biofilms, using an in vivo central venous catheter model. J Infect Dis 201: 1436-1440, 2010.

22. Hawser SP and Douglas LJ: Resistance of Candida albicans biofilms to antifungal agents in vitro. Antimicrob Agents Chemother 39: 2128-2131, 1995.

23. Mukherjee PK, Chandra J, Kuhn DM and Ghannoum MA: Mechanism of fluconazole resistance in Candida albicans biofilms: phase-specific role of efflux pumps and membrane sterols. Infect Immun 71: 4333-4340, 2003.

24. Rabea EI, Badawy ME, Stevens CV, Smagghe G and Steurbaut W: Chitosan as antimicrobial agent: applications and mode of action. Biomacromolecules 4: 1457-1465, 2003.

25. Sudarshan NR, Hoover DG and Knorr D: Antibacterial action of chitosan. Food Biotechnol 6: 257-272, 1992. 